SERIES ON EDUCATION Series editors: Anthony D Woolf, Michael Doherty

\title{
Rheumatology: What should all doctors know?
}

\author{
Michael Doherty, Peter Lanyon
}

\section{Summary}

Although training (acquisition of specific skills) may be distinguished from education (an important value base, broader in concept) both are required for medical practice. Undergraduate education should adequately prepare students for their next career stage and lay the foundation upon which postgraduate training and continuing education build. For "rheumatology" the key essential competencies that all doctors should acquire as an undergraduate are: the ability through appropriate inquiry and examination to differentiate normality from abnormality within the system; accurate recognition of common locomotor conditions and locomotor emergencies; and the ability to outline appropriate investigation and management plans for common or urgent conditions. Linked to these are the understanding of applied anatomylphysiology and basic disease mechanisms, and professional and humanitarian attitudes. Most undergraduate education, therefore, needs to focus on informed holistic patient assessment.

Learning objectives need to be clearly stated in the curriculum; the most efficient learning methods then need to be employed; and an assessment that accurately reflects the learning objectives needs to be applied. Each of these three elements needs constant review in the light of learner requirement and performance. Given the primacy of patient assessment in the objectives, clinical competencies should be prominent in the teaching and assessments. Published guidelines on development of a European core curriculum should usefully act as a focus for curriculum development within Europe. Each school can build additional objectives around this core, leading to appropriate diversity of interests and more specialised expertise in young doctors. Medical training from medical school entry onwards ideally should be viewed and organised as a single longitudinal integrated programme rather than as disjointed autonomous units.

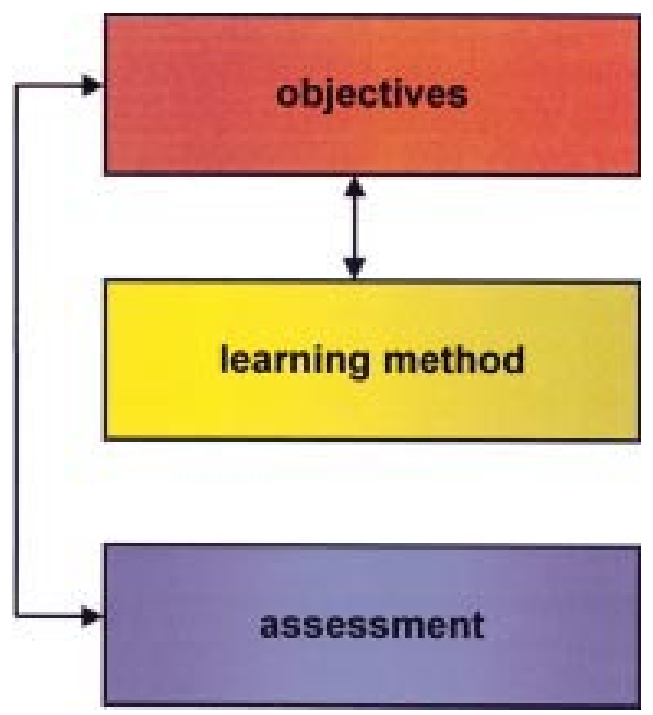

Figure 1 The three key elements of an educational programme.

Before considering the justification and content of "rheumatology" in the undergraduate core curriculum it is germane to consider:

- The key components of any educational programme and the way they interrelate

- The long term aims and objectives of medical education and training.

Components of an educational programme

"If you don't know where you're going you probably ain't going to get there"

FOREST GUMP

\section{CURRICULUM}

When planning any educational programme the crucial starting point is the curriculum (fig 1). This is where the aims and purpose of the programme are specified, with description of the skills, attitudes, and knowledge (that is, the behavioural change) that the learner will acquire by successfully moving through the programme. The aims briefly describe in general terms the content and direction of learning but are often too general to give a real guide to the level of competence that will be acquired. By contrast, the objectives of the programme and its various components (from courses or modules down to individual teaching sessions) detail the
Accepted for publication 7 March 2000 


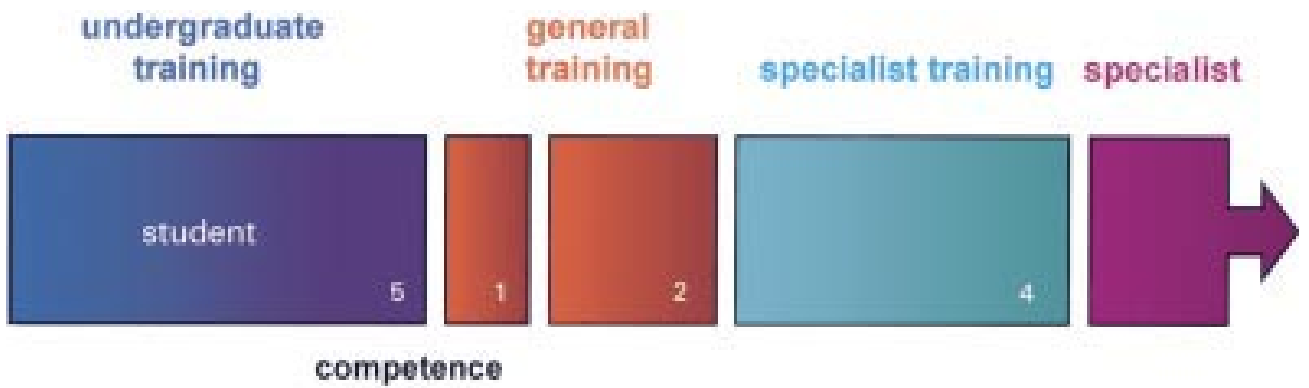

performance

Figure 2 The usual stages in education and training from medical school entry to becoming a specialist (total 12 years) and the shift from competence to performance.

more specific individual elements that must be learnt and accomplished in order to fulfil the programme aims. Such learning objectives constitute the basic fabric of the course, give a clear indication of the level of competence that should be acquired, and are sufficiently specific that whether they have been achieved or not can be readily assessed.

TEACHING METHOD

Once the curriculum is agreed the next step is to choose the most appropriate and efficient teaching method that facilitates the learning of the objectives. Clearly, different methods will be appropriate for the assimilation of factual knowledge compared with learning a clinical skill, or acquiring a professional and ethical attitude to a patient problem. In a large programme it is therefore inevitable that a variety of teaching methods are employed. Ideally, all methods should themselves be evidence based. Apart from theoretical considerations, the choice of methods will be influenced by practical constraints, such as the teaching facilities, the teacher/facilitator resource, the facilitator:learner ratio, or the availability of patients for teaching purposes. In general, however, learning is most efficient when the learner knows from the outset the specific learning objectives, when the learner plays an active part in the learning process, and when the learning is problem based. ${ }^{12}$ Reinforcement at several, rather than just one, time point is important for long term retention.

ASSESSMENT

The third major component of an educational programme is assessment. This primarily determines whether the learning objectives have been achieved. It is therefore important, though not always apparent in practice, that the assessment should clearly relate to, and reflect, the learning objectives. An appropriate assessment is the principal way in which the success of a course is audited. Assessment, however, often has other functions. For example:

- When accompanied by detailed feedback it may itself prove a useful learning experience (formative assessment)

- If graded, rather than pass/fail, it permits comparison of performance between peers
- It often has a "gate-keeping" function to prevent poor students from progressing in the course

- It may prove an incentive to learn ("the stick of assessment").

Once in place, the programme requires regular audit and quality control. Readjustment and change should be undertaken to ensure that the curriculum remains relevant and that the course is as efficient and educationally sound as possible.

\section{Longitudinal perspective of medical education}

For any profession, including medicine, learning is life long. Although training (acquisition of specific skills) may be distinguished from education (an important value base, broader in concept), it is recognised that both are important for medical practice. The content and design of a medical training programme need to take into account:

- What has gone before-the attitudes, knowledge, competencies, and maturity of the incoming learner

- What follows afterwards - both the immediate requirements for the next career stage and the long term requirements of the independent professional.

Medical school entry heralds the start of medical training following the broad education and basic science studies undertaken at school. Medical studentship is usually five years (fig 2) and leads into a period of supervised apprenticeship in a hospital (usually one junior and two senior "house officer" years). This, in turn, is followed by more focused in depth specialist "registrar" training (four years for most medical specialties) before independent existence as a qualified doctor with specified accreditation (general practitioner, doctor, or surgeon with a specialty interest, etc). Even as a specialist, continuing education and updating is required to maintain professional standards, with lifelong learning being one of the key components of clinical governance.

In an ideal world this lengthy medical training would be fully integrated. The developing doctor would pass from one year to another well prepared for each and continually rein- 
forcing and building on the professional attitudes, skills, and knowledge required for the final status of independent competent practitioner. As students enter medical school and progress towards independent status there is often a need to shift from predominantly teacher led, didactic teaching to more student led, self directed learning. Both forms should be predominantly problem based, encouraging an inquiring deep learning with high retention rather than a more superficial rote learning with poor retention. ${ }^{1}$ School leavers increasingly are more self directed in their approach to learning and more competent at using information technology, and this change needs to be taken into account by medical school teachers. A further change as the "student" progresses to "young doctor" should be a shift in emphasis from assessment of competency (can they do it, do they know what to do?) to assessment of performance (do they do it appropriately).

Unfortunately, rather than an integrated continuum the progressions from medical school entry onwards are often viewed as unconnected, discrete, separate stages of learning, organised and overseen by different faculties and organisations. Even within the medical school there may be sharp division between the first "preclinical" and subsequent "clinical" years, resulting in even greater partitioning and fragmentation of the learning programme. This lack of a single planned continuum is often compounded by further deficiencies in educational method. For example, suboptimal learning structures with unspecified objectives, and absent or inappropriate assessments, especially in the clinical training (post medical school) years. Absence of an integrated longitudinal training strategy often results in:

- Lack of understanding of learner competence and requirements at entry into each "stage"

- Less than appropriate preparation for the next "stage"

- Lack of reinforcement of important attitudes and core competencies at multiple time points

- Stress and feelings of inadequacy in junior doctors when they begin to assume responsibilities for which they feel ill prepared.

However, attempts to correct this situation are underway. For example, in our own school in Nottingham the first house officer year is now viewed as the sixth medical school year; there is a specific "preparation for house officer" transition course; and longitudinal themes such as "personal and professional development" continue throughout all six years. The introduction of specified learning objectives and regular documented appraisals with a "professional portfolio" for all the clinical postgraduate training years, and the greater coordination (and planned eventual unification) of undergraduate and postgraduate supervisory offices should also improve longitudinal integration. However, many of us still have a long way to go.
Case for "rheumatology" in the undergraduate curriculum

There are cogent arguments for inclusion of the musculoskeletal system and its afflictions in the core curriculum for medical training. For example:

- Musculoskeletal pain and disability are common and present a large community burden

- Ability to assess the locomotor system is a common requirement for many practising doctors

- Many facets of rheumatic conditions illustrate generic principles, and require skills and attitudes that are pertinent to medicine and patient care in general

- Conditions that affect the locomotor system offer excellent, common models for a variety of core curriculum topics (for example, pathogenic mechanisms, chronic disability, therapeutics, rehabilitation, primary disease prevention).

HOW MUCH AND WHEN?

The more contentious issues are how much and when to include learning of musculoskeletal disorders. For example, it is a common error to attach the above justifications to "rheumatology" or "orthopaedics" and to focus most locomotor teaching on multisystem inflammatory disease and surgical procedures rather than on the simple competencies and common conditions on which the justifications are based. Teaching of basic core competencies is thus often sacrificed for teaching that is more appropriate for specialist trainees. Although it may be delivered with enthusiasm, it comes too early, reflecting the bias and interest of the hospital based specialist rather than the requirement of the undergraduate.

With respect to how much, guidelines for a rheumatology undergraduate core curriculum were published in 1999 by the EULAR Standing Committee on Education and Training. ${ }^{3}$ This followed and extended previous guidelines that described the aims, but not specific learning objectives, and principles of development of such a core curriculum. ${ }^{4-6}$ The 1999 guidelines are intended as a discussion document and the committee will continually audit, modify, and republish the recommended curriculum over future years. The general aims of the core curriculum are stated as follows:

"The major emphasis of an undergraduate "rheumatology" course should be on clinical skills and competencies relating to the musculoskeletal system". At the end of the course students should be able to assess, by appropriate history and examination in a problem-oriented approach, an adult patient with locomotor symptoms in terms of:

- Descriptive abnormality/impairment

- Disability and handicap

- State a limited differential diagnosis

- State relevant investigations

- Outline an appropriate management (medical, surgical, rehabilitation) plan.”

The specific learning objectives are then listed under three sections: (a) competencies in clinical assessment and diagnosis; (b) knowledge of main characteristics and principles of 
management and rehabilitation of specific conditions; and (c) core knowledge, supporting diagnosis and management. The complete list of objectives is too long to repeat here, but the following are examples from each section to show the degree of definition achieved:

"- To differentiate by patient inquiry, examination, and limited investigation, common mechanical back pain ( \pm root entrapment), inflammatory back pain (for example, spondylitis), and destructive back pain (malignancy, sepsis)

- To (1) specify the symptoms, signs, and predisposing factors, and (2) outline the investigation and management of septic arthritis and bone infection

- To outline the relative prevalence, major associations (age, sex, genetic, constitutional, environmental, occupational), and expected prognosis/ outcome of neck and back pain, mechanical soft tissue lesions, osteoarthritis, osteoporosis, common fractures, rheumatoid arthritis, seronegative spondyloarthropathies, crystal associated arthritis (urate, pyrophosphate), fibromyalgia, polymyalgia rheumatica, and connective tissue diseases."

A distillation from the 1999 guidelines of the essential musculoskeletal competencies that all doctors should acquire as an undergraduate might be (a) the ability through appropriate inquiry and examination to differentiate normality from abnormality within the system; (b) accurate recognition of common locomotor conditions and locomotor emergencies; and (c) the ability to outline appropriate investigation and management plans for common or urgent conditions. Linked to these are the understanding of applied anatomy/physiology and basic disease mechanisms.

We fully endorse the suggested core competencies listed in the guidelines. However, several issues arising from the document merit further emphasis or consideration. For example:

DOGMATIC APPLICATION OF A UNIVERSAL

CURRICULUM?

It is clearly emphasised in the guidelines that development of an educational programme is a dynamic process. Adaptation of the suggested core set by individual sets of teachers is expected and is an important formative process that facilitates teacher ownership and commitment. Furthermore, only suggested core competencies are listed. Other objectives may be added by individual schools to reflect factors such as local population or medical practice requirements. Although such diversity between schools is to be welcomed, the guidelines are encouraging consideration of a common basic standard within Europe that is appropriate for undergraduates. It is hoped that schools will not expand the set too much and result in factual overload. Throughout the guidelines it is clinical skills and "principles" and "outlines" of investigation and management that are emphasised. These are the basic foundation aspects that can be carried forward and built on and supplemented by more detailed, changing knowledge through continuing education and medical informatics.
A RHEUMATOLOGY OR MUSCULOSKELETAL CURRICULUM?

The 1999 guidelines were formulated by a committee of rheumatologists on behalf of EULAR and clearly have "rheumatology" in the title. This seems to fly against the appropriate move from specialty based to systems based learning. The reason, however, was that no orthopaedic or other related specialists (for example, general practitioners, rehabilitationalists) took part in the discussions and it would have seemed arrogant to lay claim to the whole system without input from other interested teachers. Nevertheless, the guidelines are clearly "musculoskeletal" and contain many individual objectives (for example, relating to common fractures, large joint surgery, and disability) that might be expected within "orthopaedic" or "rehabilitation" core curricula. As stated in the guidelines, it is hoped that individual rheumatology teachers will liaise with other teachers to formulate a truly integrated programme with the guidelines as a focus and catalyst for discussion.

PRIME FOCUS ON ADULTS

The major focus on adults simply reflects the epidemiology of common rheumatic disorders and the common requirements of most doctors. Some more common or urgent paediatric conditions are included but most of paediatrics, including paediatric "rheumatology", is regarded as postgraduate and outside the requirement of all doctors. In some schools, the paediatric/child health core curriculum may specify objectives that relate to the musculoskeletal system.

GENERIC PROFESSIONAL OBJECTIVES

The guidelines omit specification of generic attitudes and skills that all young doctors should acquire, for example professional and humanitarian attitudes and the ability to communicate effectively with patients and healthcare professionals. Although it is assumed that such universally approved aims will exist elsewhere in any medical school curriculum, it is often hard to identify where such vital components are being learnt. We strongly support inclusion of specific objectives relating to professional and humanitarian attitudes within any locomotor module. The assessment and management of anyone with musculoskeletal pain or disability requires a professional, informed holistic approach and this should be explicit in all learning related to the system.

TIMING AND METHOD OF DELIVERY

The timing of musculoskeletal learning will clearly depend on a number of factors relating to the overall structure of the five to six year curriculum. Ideally, however, elements of the core curriculum should be spread throughout the course rather than being focused within a single module. Each successive increment can then build on the previous learning experience, giving greater retention through successful vertical integration. For example, the "GALS" locomotor screen ${ }^{7}$ is usefully learnt and applied early in the clinical course, acting not only as an 
easily learnt method to determine abnormality and disability within the system but also as a sound foundation for the later learning of more detailed regional examination.

The method of teaching will also vary according to local factors, such as teacher availability and access to suitable patients. However, given that appropriate and accurate patient assessment is central to the core objectives, teaching should predominantly focus on history and examination skills and clinical competencies related to common or urgent locomotor conditions. Patient inquiry and examination should also be prominent in student assessments. This means that clinical skills teaching and patient assessment should be more prominent in the timetable than lectures, and that clinical skills assessment (for example, using OSCEs-objective structured clinical examinations ${ }^{89}$ or OSLERs-objective structured long examination records ${ }^{10}$ ) should feature as much if not more than MCQs (multiple choice questions).

It is apparent that many of these core objectives can be taught by teachers other than rheumatologists or orthopaedic surgeons. Indeed, a good case can be made for basing much of the learning in general practice and in the community where the vast majority of musculoskeletal healthcare delivery occurs. Especially in schools with expanding student numbers, a shift of the clinical teaching base from traditional secondary/tertiary hospitals to smaller, satellite hospitals, general practices, and community seems inevitable. Such dispersed "virtual medical schools" can often offer improved teacher:learner ratios (often $1: 1$ ), easier patient access, and exposure to a more representative spectrum (of both diagnosis and severity) of musculoskeletal disorders. Clearly, a shift towards greater community based locomotor teaching needs to be supported by an increase in the teaching resources in this setting. Traditionally, a lack of rheumatology training among general practitioners themselves has been viewed as a potential obstacle to such initiatives. ${ }^{11}$ However, this situation is rapidly changing owing both to improved undergraduate teaching and specific postgraduate activities by groups such as the Primary Care Rheumatology Society in the UK.

\section{Conclusion}

Suggested core competencies relating to the musculoskeletal system that all doctors should possess have been proposed. These need discussion and modification within each medical school and will be continually reviewed for Europe as a whole. The implications are that undergraduate clinical teaching and assessment should predominantly focus on clinical history and examination skills, informed holistic patient assessment, and clinical competencies relating to common or urgent locomotor conditions. The placement of such basic competencies within the first five years of medical education/training is appropriate for continuing medical education.

1 Coles CR. Differences between conventional and problembased curricula in their students' approach to studying. Med Educ 1985;19:308-9.

2 Walton HJ, Matthews MB. Essentials of problem-based learning. Med Educ 1989;23:542-58.

3 Doherty M, Woolf A. Guidelines for rheumatology core curriculum. Ann Rheum Dis 1999;58:133-5.

4 Bjelle A. EULAR and WHO issue undergraduate curriculum guidelines to enhance clinical competence in rheumatology throughout Europe. PANLAR Bulletin 1991;6:611.

5 Guidelines on educational rheumatology programmes for medical students. WHO EURO, 1992.

6 Doherty M, Dawes P. Guidelines on undergraduate curriculum in the UK. Br J Rheumatol 1992;31:409-12

7 Doherty M, Dacre J, Dieppe PA, Snaith M. The "GALS" locomotor screen. Ann Rheum Dis 1992;51:1165-9.

8 Harden RM, Gleeson FA. Assessment of clinical compeHarden RM, Gleeson FA. Assessment of clinical compe-
tence using an objective structured clinical examination tence using an objective structured
(OSCE). Med Educ 1979;13:41-54.

9 Petrusa ER, Blackwell TA, Ainsworth MA. Reliability and validity of an objective structured clinical examination for assessing the clinical performance of residents. Arch Intern Med 1990;150:573-7.

10 Gleeson F. Assessment of clinical competence using the Objective Structured Long Examination Record (OSLER). Medical Teacher 1997;19:7-14.

11 Lanyon P, Pope D, Croft P. Rheumatology education and management skills in general practice: a national study of trainees. Ann Rheum Dis 1995;54:735-73. 